\title{
An Investigation into the Prediction of the Plasma Concentration- Time Profile and Its Interindividual Variability for a Range of Flavin-Containing Monooxygenase Substrates Using a Physiologically Based Pharmacokinetic Modeling Approach $\$$
}

\author{
Venkatesh Pilla Reddy, Barry C. Jones, Nicola Colclough, Abhishek Srivastava, Joanne Wilson, \\ and Danxi Li
}

Departments of Modelling and Simulation, Oncology Drug Metabolism and Pharmacokinetics (V.P.R.), Departments of Drug Metabolism and Pharmacokinetics and Oncology (B.C.J., N.C., J.W.), and Department of Drug Safety and Metabolism (A.S.), IMED Biotech Unit, AstraZeneca, Cambridge, United Kingdom; and Pharmaron, Beijing, China (D.L.)

Received January 25, 2018; accepted June 7, 2018

\begin{abstract}
Our recent paper demonstrated the ability to predict in vivo clearance of flavin-containing monooxygenase (FMO) drug substrates using in vitro human hepatocyte and human liver microsomal intrinsic clearance with standard scaling approaches. In this paper, we apply a physiologically based pharmacokinetic (PBPK) modeling and simulation approach (M\&S) to predict the clearance, area under the curve (AUC), and $C_{\max }$ values together with the plasma profile of a range of drugs from the original study. The human physiologic parameters for FMO, such as enzyme abundance in liver, kidney, and gut, were derived from in vitro data and clinical pharmacogenetics studies. The drugs investigated include itopride, benzydamine, tozasertib, tamoxifen, moclobemide, imipramine, clozapine, ranitidine, and olanzapine. The fraction metabolized by FMO for these drugs ranged from $21 \%$ to $96 \%$. The developed PBPK models were verified with data from multiple clinical studies. An attempt was
\end{abstract}

made to estimate the scaling factor for recombinant FMO (rFMO) using a parameter estimation approach and automated sensitivity analysis within the PBPK platform. Simulated oral clearance using in vitro hepatocyte data and associated extrahepatic FMO data predicts the observed in vivo plasma concentration profile reasonably well and predicts the AUC for all of the FMO substrates within 2-fold of the observed clinical data; seven of the nine compounds fell within 2-fold when human liver microsomal data were used. rFMO overpredicted the AUC by approximately 2.5 -fold for three of the nine compounds. Applying a calculated intersystem extrapolation scalar or tissue-specific scalar for the rFMO data resulted in better prediction of clinical data. The PBPK M\&S results from this study demonstrate that human hepatocytes and human liver microsomes can be used along with our standard scaling approaches to predict human in vivo pharmacokinetic parameters for FMO substrates.

\section{Introduction}

Flavin-containing monooxygenase (FMO) belongs to a family of enzymes that oxygenate a wide variety of nucleophilic heteroatomcontaining chemicals and drugs. FMO3 is the predominant functional isoform in adult human liver, while FMO1 and 5 are expressed in adult kidney and liver, respectively (Yeung et al., 2000). FMO enzymes can be differentiated from cytochrome P450 (P450) enzymes via heat inactivation and selective substrate inhibition by methimazole (Cashman and Zhang, 2006; Taniguchi-Takizawa et al., 2015). There is a lack of validated methods for extrapolating in vitro hepatic intrinsic clearance $\left(\mathrm{CL}_{\text {int }}\right)$ for FMO to in vitro/in vivo extrapolation (IVIVE) clearance (CL). Our earlier paper (Jones et al., 2017) investigated a set of 10 literature compounds with varying degrees of FMO involvement. The compounds were profiled in in vitro assays, which defined the extent of

https://doi.org/10.1124/dmd.118.080648.

S This article has supplemental material available at dmd.aspetjournals.org.
FMO involvement based on heat inactivation and inhibition by the selective substrate methimazole. The FMO contribution varied from $21 \%$ for imipramine to $96 \%$ for itropride. In addition, these data were used to predict the unbound intrinsic clearance and compared the results with human clearance data obtained from the literature. Using standard scaling scaled methods, $70 \%$ of the compounds studied had predicted unbound intrinsic clearance within 2-fold of the observed unbound intrinsic clearance.

In this paper, we apply a mechanistic, physiologically based pharmacokinetic (PBPK) modeling and simulation approach (M\&S) to predict the observed clinical data of drugs that are metabolized by FMO. The utility of PBPK modeling via a top-down approach [typically involving the estimation of the model parameters using observed clinical pharmacokinetic (PK) profiles] for FMO was shown for itopride (Zhou et al., 2017), which evaluated the impact of FMO3 polymorphism on exposure in Asian subjects. This study showed that genotyping for FMO3 to exclude subjects with homozygous Lys158/Gly308 alleles is not clinically relevant in terms of exposure, and thus is not required.

ABBREVIATIONS: ASA, automated sensitivity analysis; $\mathrm{AUC}$, area under the curve; $\mathrm{CL}$, clearance; $\mathrm{CL}_{\text {int }}$, intrinsic clearance; $\mathrm{FMO}$, flavin-containing monooxygenase; HLM, human liver microsomes; ISEF, intersystem extrapolation factor; IVIVE, in vitro/in vivo extrapolation; M\&S, modeling and simulation approach; P450, cytochrome P450; PBPK, physiologically based pharmacokinetic; PE, parameter estimation; PK, pharmacokinetic; rFMO, recombinant FMO; UGT, UDP-glucuronosyltransferase. 
However, the elimination kinetics of itopride from in vitro studies using human liver microsome (HLM) data resulted in about 8-fold underprediction of itopride clearance (Obach et al., 1997; Humphries et al., 2015; Zhou et al., 2017). This was similar to the FMO substrate benzydamine, where underprediction was reported to be about 1.5- to 11-fold (Obach et al., 1997; Humphries et al., 2015; Zhou et al., 2017). Thus, an additional investigation to achieve a better in vitro/in vivo extrapolation was warranted. As a result of the aforementioned observations, Jones et al. (2017) generated in vitro data under welldefined experimental conditions. To date, there has not been a comprehensive assessment of FMO scaling using in vitro data and a bottom-up PBPK M\&S approach (which typically uses in vitro data along with mechanistic understanding of absorption, distribution, metabolism, and excretion processes of a drug to prospectively simulate the PK profiles) for substrates with differing FMO contributions.

A human PBPK model, once established, can be a valuable tool to inform the clinical study design of an investigational drug under development, and help to link the exposure from healthy subjects to patients by taking into account pharmacogenetics and extrinsic factors or simulating the PK profiles in special populations such as pediatric subjects. Hence, the aim of this study was to investigate the performance of PBPK M\&S to predict the concentration-time profiles, PK parameters [area under the curve (AUC), $C_{\max }$ and CL], and interindividual variability for a set of compounds for which all or part of the in vivo clearance has been determined to be mediated by FMO.

\section{Materials and Methods}

\section{Compound Selection and Clinical Data Collection}

Nine orally administered drugs, namely, itopride, benzydamine, tozasertib, tamoxifen, moclobemide, imipramine, clozapine, olanzapine, and ranitidine were selected for PBPK modeling based on the availability of the in vitro and in vivo data within AstraZeneca or in the public domain. Clinical plasma profiles were digitized using an AstraZeneca internal tool namely, GI-SIM (Sjögren et al., 2013), if literature data were used. The detailed clinical trial information for each of the nine drugs is described in Table 1 . The PK data and parameters for these drugs were either extracted from the literature or obtained from internal clinical studies (e.g., tamoxifen).

\section{PBPK Modeling Approach}

Whole-body PBPK M\&S of clinical PK data was performed using the population-based absorption, distribution, metabolism, and excretion simulator, Simcyp version 16, release 1 (Certara, Sheffield, United Kingdom) (Jamei et al., 2013). The modeling assumptions and parameters were verified using clinical data for the nine FMO substrates.

\section{PBPK Modeling Input Parameters}

System-Related Parameters. Among the five FMOs, only FMO1, 3, and 5 are functional in human. FMO1 is a major form in human kidney (Yeung et al., 2000), while FMO3 and FMO5 are expressed mainly in liver (Dolphin et al., 1996; Koukouritaki and Hines, 2005). The reported FMO1 (kidney), FMO3 (liver), and FMO5 (gut) protein abundance values of 47,71 , and $22 \mathrm{pmol} / \mathrm{mg}$ protein were used in this analysis along with the reported coefficient of variance (Haining et al., 1997; Overby et al., 1997; Yeung et al., 2000; Chen et al., 2016). Enzyme abundance, phenotype frequencies, and relative activities of FMO3 were accounted for within the virtual population of the simulator. The intrinsic catalytic activity per unit amount of FMO1 and FMO3 enzyme was assumed to be the same in healthy Caucasian, Japanese, and Chinese subjects since the available expression data pool broadly represents the Caucasian and Asian populations (Cashman and Zhang, 2006).

The Simcyp platform provides an enzyme kinetics pane for assessing the contribution to metabolic clearance in the liver, kidney, or intestine for P450 and UDP-glucuronosyltransferase (UGT) enzymes, but not yet for FMOs. However, a user-defined enzyme option, or unused UGT enzyme, within the UGT enzyme kinetic pane allows entry of recombinant FMO (rFMO) $\mathrm{CL}_{\text {int }}$ data, along with expression levels, frequency of polymorphism, and turnover rate (Reddy et al., 2010), to define the FMO enzyme to enable IVIVE.

Drug-Related Parameters. The PBPK model input parameters used are listed in Table 2. The in vitro parameters such as $\mathrm{CL}_{\text {int }}$, plasma protein binding (unbound fraction in plasma), and fraction unbound in hepatocyte incubation were experimentally measured (Jones et al., 2017); in some instances, the PBPK platform's prediction toolbox was employed to predict the drug-related properties such as the effective human permeability $\left(\mathrm{P}_{\text {eff }}\right)$ value using the mechanistic permeability model or the polar surface area/hydrogen bond donor model if permeability data were not available. The first-order absorption rate constant and

TABLE 1

Clinical data used for PBPK model verification

\begin{tabular}{|c|c|c|c|c|c|c|c|}
\hline Drug & $\begin{array}{c}\text { Age } \\
\text { Range }\end{array}$ & $\begin{array}{l}\text { Number } \\
\text { of Subjects }\end{array}$ & $\begin{array}{l}\text { Male/Female } \\
\text { Numbers }\end{array}$ & Dose (Route) & $\begin{array}{l}\text { Dosage Regimen/PK } \\
\text { Duration }\end{array}$ & Comments & Reference \\
\hline & $y r$ & & & & & & \\
\hline Benzydamine & $41-51$ & 6 & $6 / 0$ & $5 \mathrm{mg}$ (i.v.) & Single/48 hours & Caucasian healthy volunteers & Humphries et al. (2015) \\
\hline Benzydamine & $18-51$ & 12 & $6 / 6$ & $50 \mathrm{mg}$ (oral) & Single/48 hours & Caucasian healthy volunteers & Humphries et al. (2015) \\
\hline Itopride & - & 6 & $6 / 0$ & $50 \mathrm{mg}$ (oral) & Single/24 hours & Japanese healthy volunteers & Yoon et al. (2014) \\
\hline Itopride & $24-31$ & 5 & $5 / 0$ & $150 \mathrm{mg}$ (oral) & Single/24 hours & Japanese healthy volunteers & Katagiri et al. (2006) \\
\hline Tozasertib & $22-80$ & 27 & $14 / 13$ & $\begin{array}{c}4,8,16,32,45,64, \text { and } \\
96 \mathrm{mg} / \mathrm{m}^{2} \text { (i.v.) }\end{array}$ & $\begin{array}{l}\text { Single, 24-hour } \\
\text { infusion }\end{array}$ & Oncology patients & Traynor et al. (2011) \\
\hline Tamoxifen & $29-71$ & 29 & $4 / 25$ & $20 \mathrm{mg}$ (oral) & Multiple, PK at SS & Oncology patients & AstraZeneca internal data ${ }^{a}$ \\
\hline Tamoxifen & $41-64$ & 24 & $0 / 24$ & $30 \mathrm{mg}$ (oral) & Single & Healthy volunteers & Fuchs et al. (1996) \\
\hline Moclobemide & $21-30$ & 12 & $12 / 0$ & $\begin{array}{l}150 \mathrm{mg} \text { (i.v.), } \\
20 \text { minute infusion }\end{array}$ & Single & Healthy volunteers & Raaflaub et al. (1984) \\
\hline Moclobemide & $21-30$ & 12 & $12 / 0$ & $100 \mathrm{mg}$ (oral) & Single & Healthy volunteers & Schoerlin et al. (1987) \\
\hline Imipramine & $23-64$ & 8 & $8 / 0$ & $100 \mathrm{mg}$ (oral) & Single & Caucasian healthy volunteers & Albers et al. (2000) \\
\hline Imipramine & $22-37$ & 11 & $5 / 6$ & $50 \mathrm{mg}$ (i.v.) and $100 \mathrm{mg}$ (oral) & Single & Caucasian healthy volunteers & Brøsen and Gram (1988) \\
\hline Clozapine & $21-30$ & 18 & $18 / 0$ & $100 \mathrm{mg}$ (oral); twice daily & Single & Asian healthy volunteers & Tassaneeyakul et al. (2005) \\
\hline Clozapine & $30-32$ & 2 & $2 / 0$ & 200 and $600 \mathrm{mg}$ (oral) & Multiple, PK at SS & Caucasian schizophrenic patients & Takano et al. (2006) \\
\hline Olanzapine & $28-50$ & 10 & $10 / 0$ & $10 \mathrm{mg}$, oral & Single & Schizophrenic patients & Elshafeey et al. (2009) \\
\hline Olanzapine & $19-41$ & 24 & $24 / 0$ & $10 \mathrm{mg}$ (oral) & Single & Healthy volunteers & Chiu et al. (2004) \\
\hline Ranitidine & $21-23$ & 6 & $6 / 0$ & $20 \mathrm{mg}$ (i.v.) and $100 \mathrm{mg}$ (oral) & Single & Healthy volunteers & McNeil et al. (1981) \\
\hline Ranitidine & $19-32$ & 12 & $12 / 0$ & $100-400 \mathrm{mg}$ (oral) & Single and multiple & Healthy volunteers & Garg et al. (1985) \\
\hline
\end{tabular}

SS, steady-state. - , data not available or not reported.

${ }^{a}$ Clinical trial NCT02093351: To assess safety and effect of olaparib on the pharmacokinetics of anastrozole, letrozole, and tamoxifen, and their effect on olaparib, in patients with advanced solid cancer (2017). 


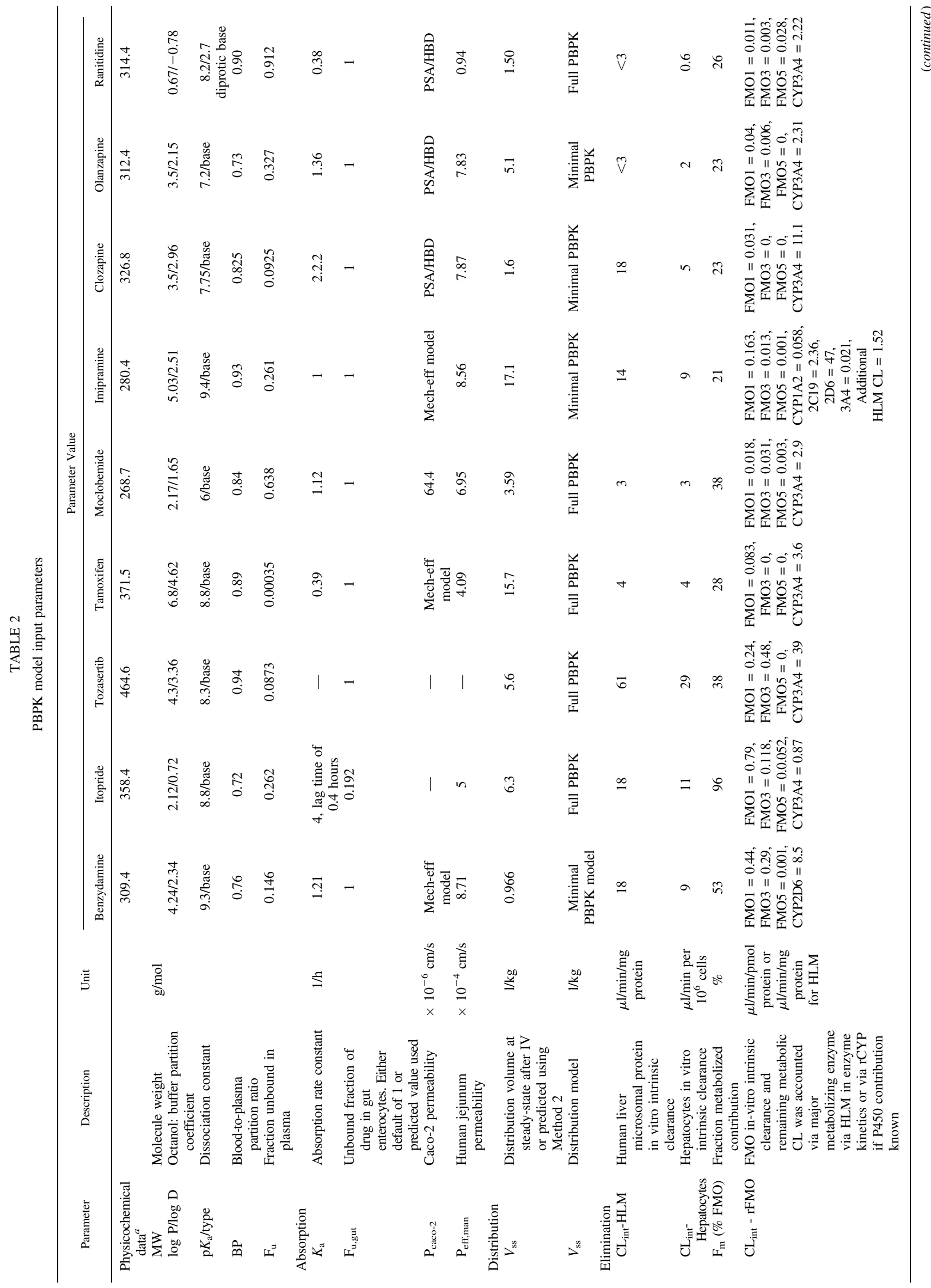


fraction absorbed were estimated from clinical data or predicted based on the $\mathrm{P}_{\mathrm{ef}}$ value within the Simcyp simulator. Unless otherwise specified, the volume of distribution at steady state for all compounds was predicted using the Rodgers and Rowland method (Rodgers et al., 2005; Rodgers and Rowland, 2006) within the PBPK simulator. The fraction unbound in microsomes and hepatocytes (and fraction unbound in hepatocyte incubation) was also included in the model.

Intrinsic Clearance Measurement Using Hepatocytes and Human Liver Microsomes. The measured in vitro data are detailed in Supplemental Table 1, and for methodology refer to Jones et al. (2017). The assignment of FMO metabolic intrinsic clearance and methodology employed to assess drug elimination are given Supplemental Table 2.

Intrinsic Clearance Measurement in rFMO1, rFMO3, and rFMO5. The compounds (final concentration $1 \mu \mathrm{M}$ ) were incubated with $\mathrm{rFMO} 1, \mathrm{rFMO} 3$, and rFMO5 (final concentration $0.5 \mathrm{mg} / \mathrm{ml}$ protein). Incubations were carried out in duplicate in $100 \mathrm{mM}$ potassium phosphate buffer ( $\mathrm{pH}$ 7.4) containing NADPH $(1 \mathrm{mM})$ in a total volume of $0.2 \mathrm{ml}$. Incubations were commenced with the addition of NADPH and carried out in a 96-well plate at a temperature of $37^{\circ} \mathrm{C}$. At times ranging between 0 and 50 minutes, aliquots $(0.02 \mathrm{ml})$ were removed and added to $0.08 \mathrm{ml}$ of termination solution (acetonitrile + formic acid) containing buspirone ( $4 \mathrm{nM})$ as an internal standard. Samples were vortexed and centrifuged at $3500 \mathrm{rpm}$ for 5 minutes, and the resulting supernatant was collected for liquid chromatography-tandem mass spectrometry analysis as shown in the Supplemental Material and Supplemental Table 5.

Estimating the Tissue-Specific Scalar or Intersystem Extrapolation Factor for Extrapolation Using rFMO Data. Human PK predictions using PBPK models and in vitro assays such as hepatocytes or HLM data combined with the appropriate scalars appears to be robust for P450 enzymes (Wagner et al., 2015; Luzon et al., 2017). However, very limited knowledge of scaling factors exists for drugs that are metabolized by non-P450s such as FMOs. A drug-specific intersystem extrapolation factor (ISEF) value can be derived using the following formula (see Supplemental Table 1):

$\mathrm{ISEF}=\frac{\left[\mathrm{CL}_{\text {int,u }} \text { in HLM }(\mu \mathrm{l} / \mathrm{min} / \mathrm{mg})\right] /[\mathrm{HLM} \text { FMO abundance }(\mathrm{pmol} / \mathrm{mg})]}{\mathrm{CL}_{\text {int,u }} \text { in } \mathrm{rFMO}(\mu \mathrm{l} / \mathrm{min} / \mathrm{pmol})}$

When developing a model based on the rFMO data, the elimination via FMO1, FMO3, and FMO5 enzymes was considered separately along with respective liver, kidney, and intestine expression levels. The intrinsic catalytic activity per unit amounts of FMO1, FMO3, and FMO5 enzymes were $71 \mathrm{pmol} / \mathrm{mg}$ protein in liver, $47 \mathrm{pmol} / \mathrm{mg}$ protein in kidney, and $22 \mathrm{pmol} / \mathrm{mg}$ protein in intestine to reflect the abundances of FMO3, FMO1, and FMO5, respectively. These abundance values were included in the population file using unused UGT enzyme with the user-defined option within Simcyp (Supplemental Table 2). The impact of FMO3 polymorphism was included in the final PBPK model. The remaining metabolic CL was accounted for via major metabolizing enzymes of the drug via HLM in enzyme kinetics or via recombinant P450 if P450 contribution was known, as shown in Table 2.

The automated sensitivity analysis (ASA) or parameter estimation (PE) option within Simcyp was used to determine the tissue-specific rFMO scalar in liver, intestine, and kidney tissue or to determine the ISEF value. To this end, we used clinical data of the drugs that are mainly metabolized by FMO such as itopride. When the PBPK model was optimized to the clinical data of itopride using the ASA approach, the lower and upper bounds provided for the rFMO scalar parameter were $0-100$ and the used log-distributed step size with 10 steps (increasing with increased parameter value) within the Simcyp PBPK platform. The effect of the rFMO scalar on the $C_{\max }$, AUC, CL, and PK profiles were reviewed to understand the elimination trends of a drug. In addition to ASA, PE was performed using the weighted least-squares objective function to allow fitting to the mean profile. Weighting by the reciprocal of the prediction was used since this provides a good fit for both $C_{\max }$ and the terminal phase when oral data are fitted to the model. The Nelder-Mead fitting algorithm was used with the default settings (reflection coefficient $=1$, expansion coefficient $=2$, contraction coefficient $=0.5$, and shrink coefficient $=0.5$ ).

PBPK Model Verification. PK simulations were conducted during the PBPK model development as a verification step to ensure appropriate model input parameters were used. The predicted PK parameters and simulated concentrationtime profiles were compared with those observed in clinical trials (Table 1). The 

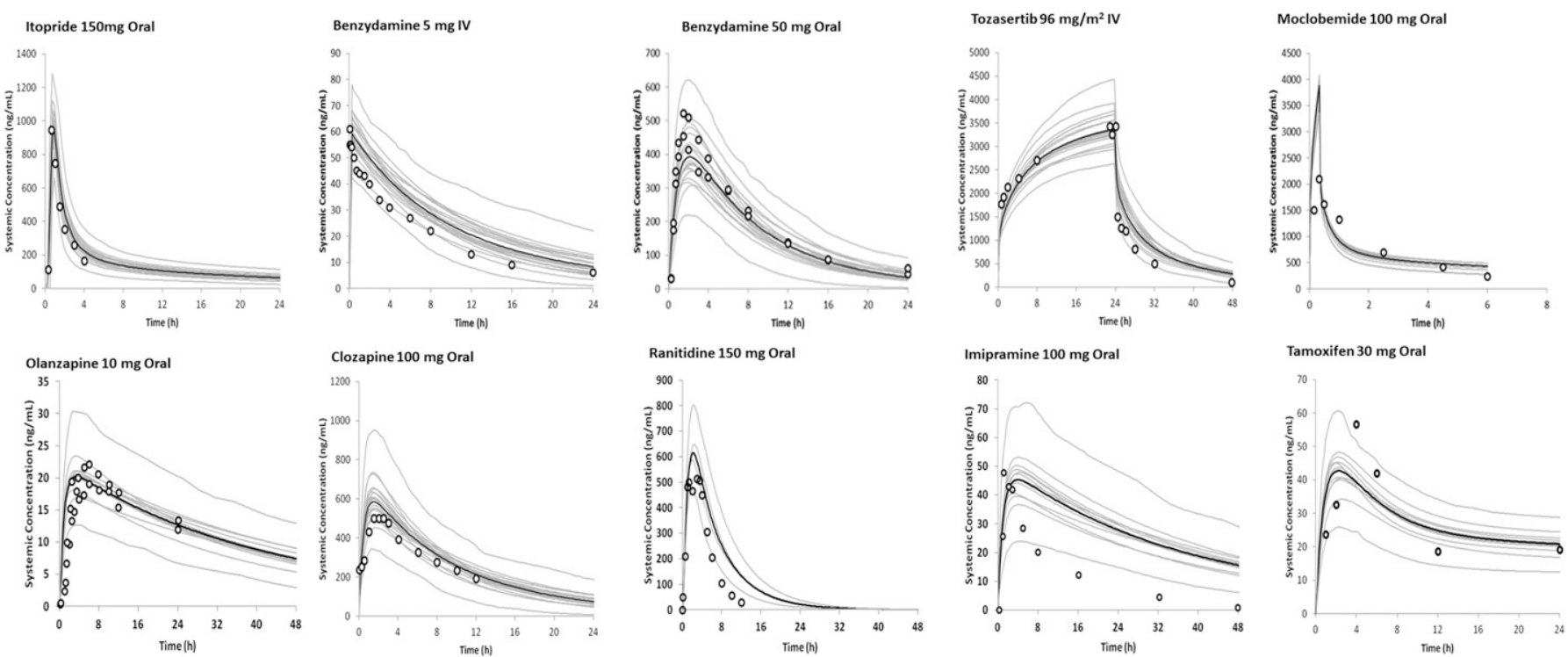

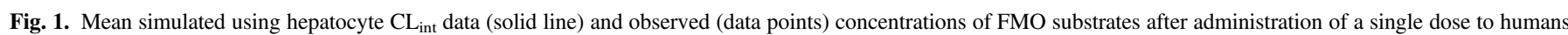

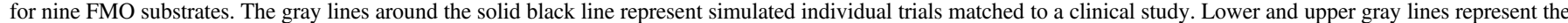
$90 \%$ confidence interval of the respective simulations.

same trial designs as the clinical studies (e.g., dose, number of subjects, age, gender, population, etc.) were used in the simulations. When a specific population was not available in the Simcyp simulator, for example, the Korean population, an alternative validated population such as the Japanese population was used instead. In the case of olanzapine and clozapine, we assumed no difference between healthy subjects and patients since no validated schizophrenic population was available.

In addition to verification of the PK parameters and PK profiles, we also investigated the nature of the correlation between human in vitro and in vivo clearance predicted by the PBPK approach using hepatocyte, HLM, and rFMO data with ISEF and tissue-specific scalars.

\section{Results}

PBPK Model Development and Optimization of PBPK Models. The plasma concentration profiles for nine orally or intravenously administrated FMO substrates were simulated by PBPK modeling using the hepatocyte $\mathrm{CL}_{\text {int }}$ data and were compared with those observed clinically, as shown in Fig. 1. By visual inspection, the developed PBPK models adequately simulated the observed clinical data, and thus it was assumed that the models reflect the metabolic routes of elimination based on in vitro hepatocyte or HLM data (Fig. 2), along with any known additional pathways such as renal or biliary clearance. The observed and model-simulated PK parameters such as AUC, $C_{\max }$, and CL are shown in Table 3. Table 3 shows that using hepatocyte $\mathrm{CL}_{\text {int }}$ data the PBPK model successfully predict the AUC and $C_{\max }$ for all nine drugs within a factor of 2 compared with the clinical data. When HLM CL $\mathrm{CL}_{\text {int }}$ data were used seven of the nine drugs had these parameters correctly predicted within a factor of 2 (Fig. 2; Supplemental Table 3). Use of rFMO $\mathrm{CL}_{\text {int }}$ data over- or underpredicted the observed clinical data for five of the nine compounds as depicted in the forest plot using the AUC as an end point (Fig. 2) when no tissue-specific factor (zero value) or ISEF option was used. The drug-specific ISEF values for each of the rFMO isoforms determined using eq. 1 are shown in Supplemental Table 1.

Based on the ASA and PE analyses using itopride clinical data, an rFMO tissue scalar value of 0.2 or a compound-specific ISEF appears to be a reasonable value to obtain a good IVIVE, as shown in Fig. 3. Performance of the tozasertib PBPK model with an rFMO scalar value is shown as an example in Fig. 3. Figure 3, A-C shows the model fit to observed data without and with the ISEF or tissue scalar for itopride, while Fig. 3, D-F shows the model fit without and with ISEF or rFMO scalar for tozasertib, respectively. This generic scalar value of 0.2 was applied for each of the FMO1, FMO3, and FMO5 isoforms and for all other compounds. This optimized rFMO scalar also resulted in better

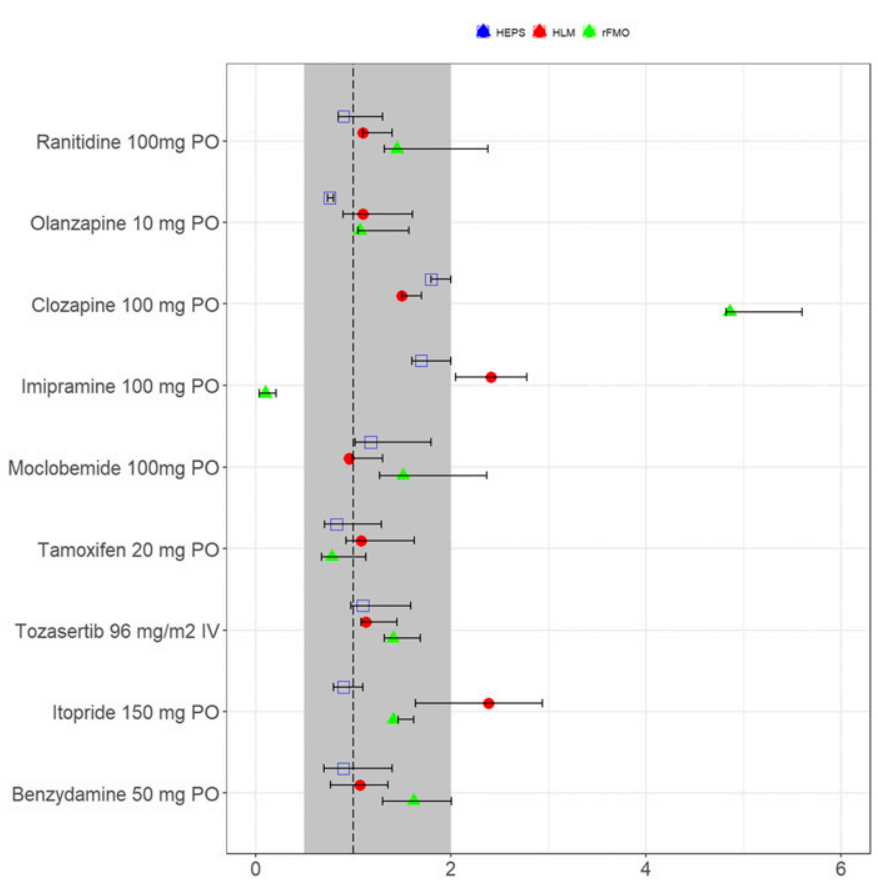

Fig. 2. Forest plot showing the PBPK modeling performance of FMO substrates. Predictions are expressed as ratios of predicted over observed AUC. The dashed line represents the identity (predicted/observed ratio), the gray shaded represents the $0.5-2.0$ ratio window, and the blue shaded area represents the 0.67 - to 1.50 -fold ratio. Mean AUC using hepatocytes (blue squares); mean AUC using HLM (red circles); mean AUC using rFMO with no scaling (green triangles), with error bars represented as percentile range (fifth and 95 th percentiles). 
TABLE 3

Observed mean PK parameters for FMO substrates using hepatocyte data

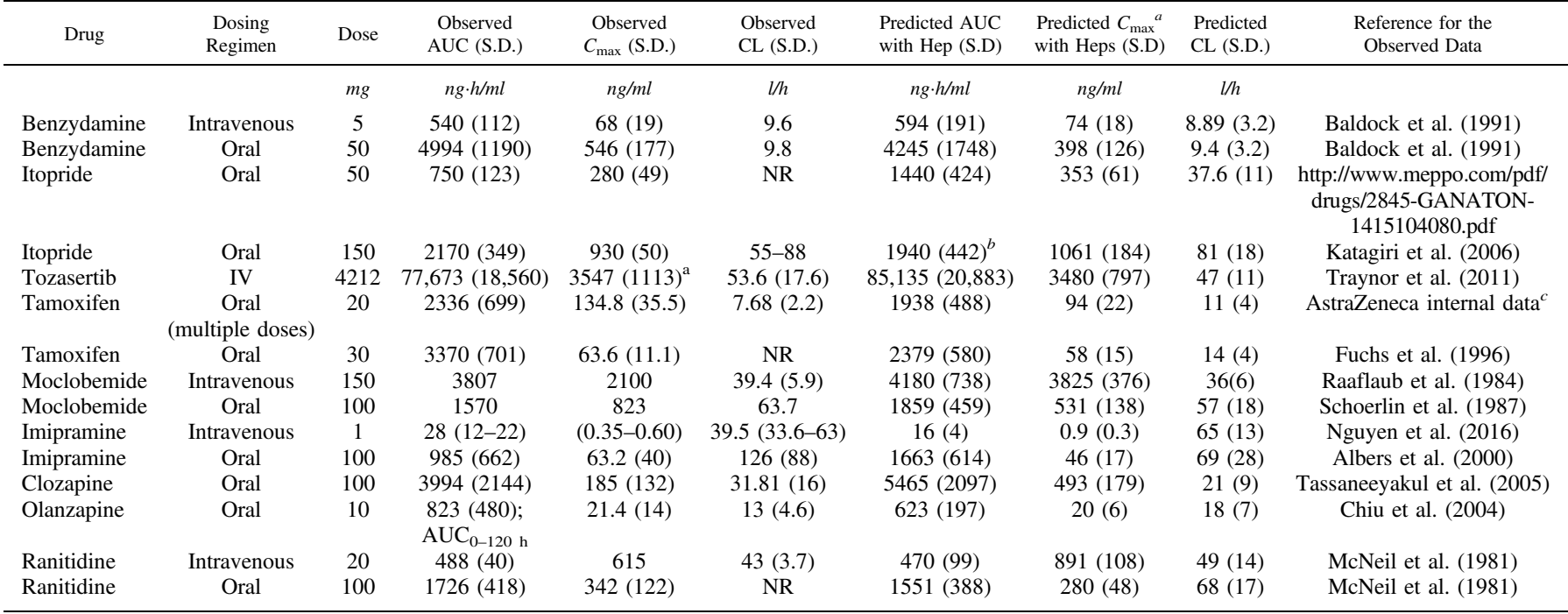

heps, hepatocytes; NR, not reported. - , data not available or not reported.

${ }^{a} C_{\max }=$ concentration at the end of infusion.

${ }^{b} \mathrm{AUC}_{0-4 \mathrm{~h}}$ to match the reported AUC.

${ }^{c}$ Clinical trial NCT02093351: To assess safety and effect of olaparib on the pharmacokinetics of anastrozole, letrozole, and tamoxifen, and their effect on olaparib, in patients with advanced solid cancer (2017).

predictions of the observed PK profiles (Fig. 3) with an increased correlation value $\left(r^{2}\right)$ for the correlation of predicted versus observed clearance, as shown in Fig. 4. The forest plot showing the model performance using rFMO data of FMO substrates with and without scalar for all compounds is summarized in Supplemental Fig. 1. The results of the PK parameters for these two options are given in Supplemental Table 3.
A Itopride $150 \mathrm{mg}$ oral without rFMO scalar

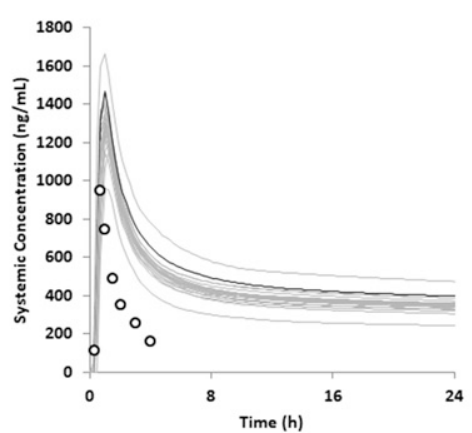

\section{Tozasertib $96 \mathrm{mg} / \mathrm{m}^{2}$ IV without rFMO scalar}

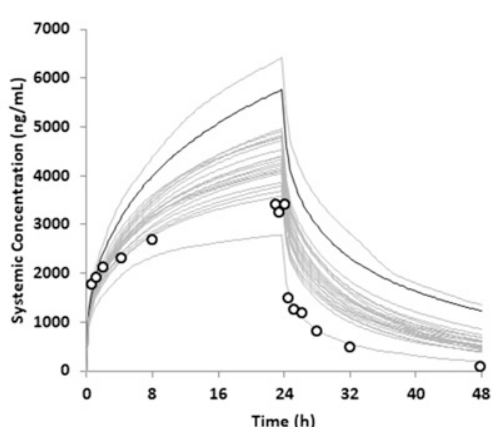

B Itopride 150mg Oral with tissue rFMO scalar

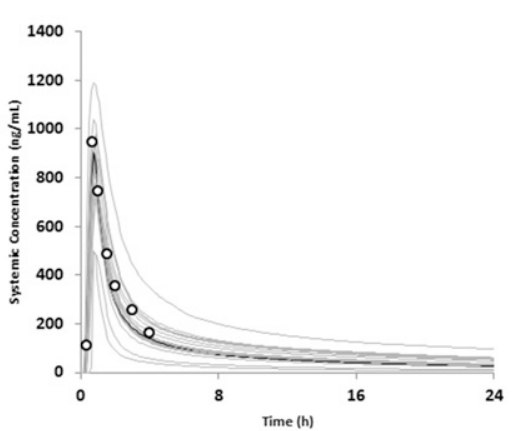

E Tozasertib $96 \mathrm{mg} / \mathrm{m}^{2} \mathrm{IV}$ with tissue $\mathrm{rFMO}$ scalar

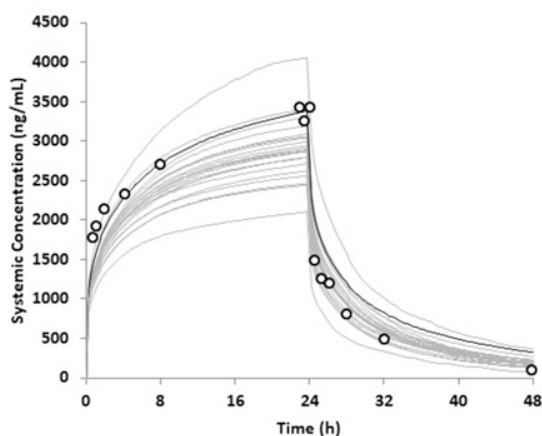

\section{Itopride 150mg Oral with rFMO ISEF scalar}

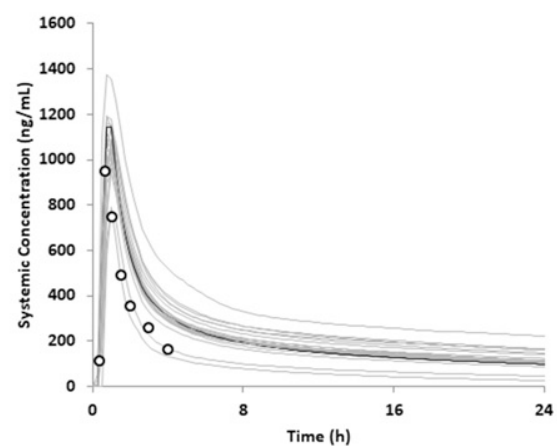

F Tozasertib $96 \mathrm{mg} / \mathrm{m}^{2} \mathrm{IV}$ with $\mathrm{rFMO}$ ISEF scalar

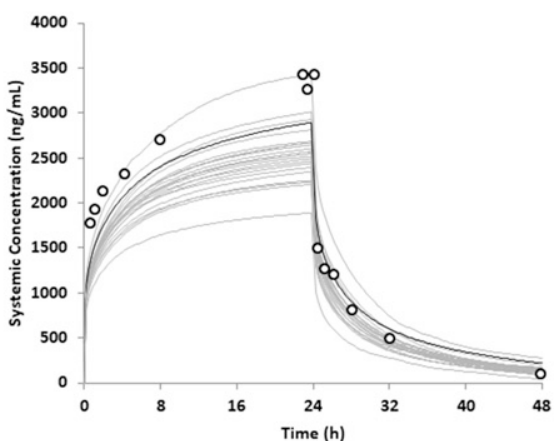

Fig. 3. Determination of rFMO scalar for itopride and its application to tozasertib as an example. (A-C) Model fit without rFMO scalar, with $\mathrm{rFMO}$ tissue scalar, and with rFMO ISEF scalar for itopride, respectively. (D-F) Tozasertib model without rFMO scalar, with rFMO tissue scalar, and with rFMO ISEF scalar, respectively. 


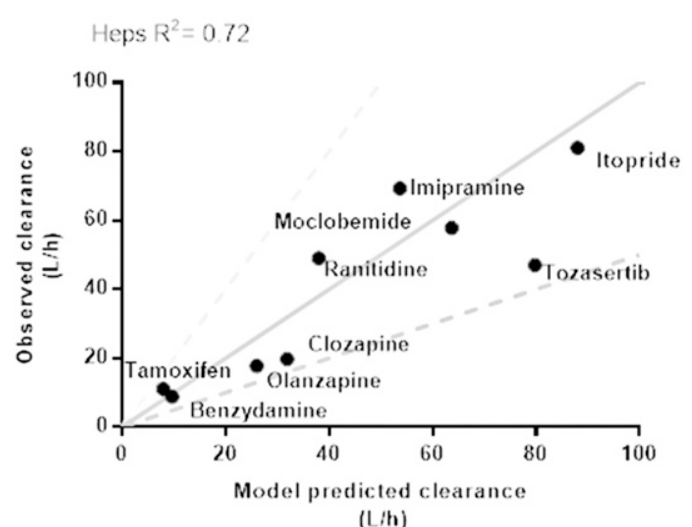

(L/h)

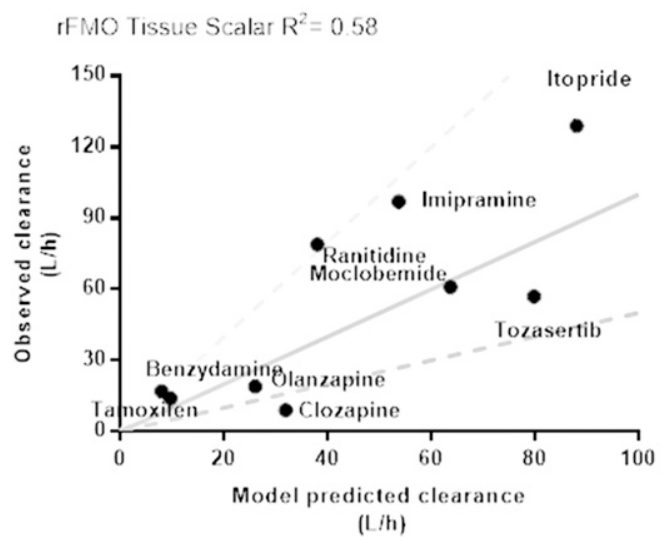

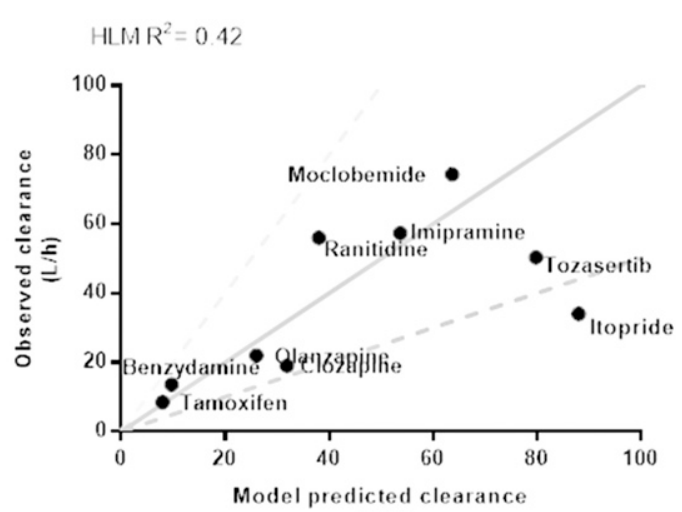

(L/h)

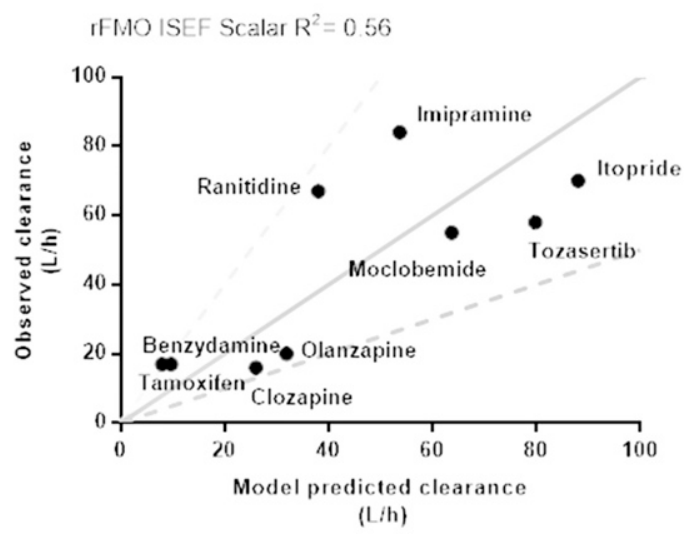

Fig. 4. Correlations for the PBPK model-predicted clearance using human hepatocytes, HLM, and rFMO with ISEF scalar and tissue-specific scalar.

\section{Discussion}

The prediction of human PK properties can be critical in decision making when ranking short listed compounds, the assessment of lead series, and for clinical drug candidates. A bottom-up PBPK modeling approach involves modeling of the mechanisms of the drug disposition processes and simulating the human PK profiles by accounting for variability arising from enzyme expression at the patient level. The application of bottom-up models depends on the availability of quality data and established IVIVE factors and scalars. Human PK predictions using PBPK models and data from in vitro assays such as hepatocytes or HLM CL $L_{\text {int }}$ combined with the appropriate scalars appears to be robust for P450 enzymes (Wagner et al., 2015; Luzon et al., 2017). However, very limited knowledge of scaling factors exists for drugs that are metabolized by non-P450s such as FMOs. As a result, early bottom-up models will require verification with in vivo clinical data, and in some cases calibration of parameters including scaling factors through a middle-out approach (i.e., combining clinical and in vitro data) to be considered as more impactful in the drug development process. A method that many consider would provide greater certainty is to compare the predictions from two or more methods. An example would be comparing two predictions based on the same input data set; in this case, comparing the results shown in our earlier study (Jones et al., 2017) versus the PBPK modeling approach.

In this paper, we use a PBPK M\&S approach to predict the PK parameters, to verify and simulate the observed clinical data of FMO substrates, and to compare the results with our earlier IVIVE results (Jones et al., 2017). Human hepatocytes produced good concordance $\left(R^{2}=0.72\right)$ between the predicted and observed in vivo clearance values (Fig. 4), while all drugs fell within 2-fold. The correlation between the predicted and observed in vivo clearance was weaker for HLM and rFMO, which is consistent with our earlier study (Jones et al., 2017).

The PBPK results using rFMO in vitro data deviated from the observed exposure by approximately 2.5 -fold, when a scalar of zero was used. This biased prediction may not be surprising since individual rFMO and HLM scaling factors are required to compensate for differences in the intrinsic activity per unit enzyme of rFMO relative to HLM. The observed clinical data can be used to refine and/or improve the model performance via a middle-out approach (combination of topdown and bottom-up approaches). We estimated a scaling factor to convert enzyme kinetic values obtained in different expression systems to the equivalent in HLM.

This rFMO scalar concept is well established for recombinant P450s within PBPK platforms such as Simcyp and has been shown to be applicable for recombinant UGTs as well (Lin, 2015). In addition to a compound-specific ISEF (Supplemental Table 1), an attempt was made to generate a tissue-specific scalar similar to that which exists for UGT enzymes within the simulator. To determine the scalar value associated with a particular rFMO isoform we performed sensitivity and PE analyses using itopride clinical data (Fig. 3). The ASA approach suggested that an rFMO scalar value of around 0.2 is required to recover the itopride clinical data. This rFMO scalar of 0.2 also resulted in better predictions for other compounds, as shown in Fig. 3 and Supplemental Fig. 1, than when the rFMO scalar of zero was used. This analysis shows that the observed data can be used to refine the model performance via fitting the clinical data to estimate a few of the model parameters (in this case, the rFMO scalar) to improve the model's predictive performance of other FMO substrates using data of a corresponding rFMO in vitro assay. 
When developing the model based on the rFMO data the elimination via FMO1, FMO3, and FMO5 was considered separately along with respective liver, kidney, and intestine expression levels. The intrinsic catalytic activity per unit amounts of FMO1, FMO3, and FMO5 enzymes were $71 \mathrm{pmol} / \mathrm{mg}$ protein in liver, $47 \mathrm{pmol} / \mathrm{mg}$ protein in kidney, and $22 \mathrm{pmol} / \mathrm{mg}$ protein in intestine to reflect the abundances of FMO3, FMO1 and FMO5, respectively, these amounts were included in the population file. The impact of FMO3 polymorphisms was included in the final PBPK model.

PBPK model approaches allow the simulation of PK profiles by taking into account parameter uncertainty. This uncertainty reflects both uncertainty in the experimental values of input parameters in a model along with variability arising from FMO expression and demographics. This is unlike simple IVIVE methods that do not take variability arising from expression, demographics, and experimental errors into account (Jones et al., 2017). Thus, the correlation values from PBPK modeling give more confidence in the scaling approach on top of simple IVIVE methods.

Once the PBPK model is verified it can be applied to predict the drug exposure differences in subjects with FMO3 wild-type and homozygous FMO Lys158/Gly308 mutant genotypes, as shown in Zhou et al. (2017) for itopride. In addition, we can apply the developed PBPK model to special populations, such as pediatrics, since FMO abundance data recently quantified will allow accounting for an ontogeny.

The ontogeny of hepatic FMO exhibits a similar trend to the CYP3A family, where FMO1 resembles the pattern of CYP3A7 and FMO1 and FMO3 expression data have been quantified using a HLM system (Koukouritaki et al., 2002). Moreover, it was shown that FMO3 matures to the adult level by 10 years of age (Hines and McCarver, 2002; Koukouritaki et al., 2002), requiring a dose adjustment because of this ontogeny process. Currently, the pediatric clinical data for FMO substrates is lacking, but a prospective simulation for itopride across the age range from 6 months to 12 years of age is shown in Supplemental Table 4.

Our results confirm that clearance by FMO in human can be assessed and derisked using a set of in vitro drug metabolism and PK assays. When combined with PBPK modeling, it allows for more robust prediction of AUC and other PK parameters further supporting derisking during the drug development process.

\section{Acknowledgments}

We thank Andy Sykes, Helen Humphries, Iain Gardner, and Sibylle Neuhofff for scientific input in carrying out the PBPK analyses described in this paper.

\section{Authorship Contributions}

Participated in research design: Pilla Reddy, Jones, Colclough.

Conducted experiments: Srivastava, Wilson, Li.

Performed data analysis: Pilla Reddy, Jones.

Wrote or contributed to the writing of the manuscript: Pilla Reddy, Jones, Srivastava, Colclough, Wilson, Li.

\section{References}

Albers LJ, Reist C, Vu RL, Fujimoto K, Ozdemir V, Helmeste D, Poland R, and Tang SW (2000) Effect of venlafaxine on imipramine metabolism. Psychiatry Res 96:235-243.

Baldock GA, Brodie RR, Chasseaud LF, Taylor T, Walmsley LM, and Catanese B (1991) Pharmacokinetics of benzydamine after intravenous, oral, and topical doses to human subjects. Biopharm Drug Dispos 12:481-492.

Brøsen K and Gram LF (1988) First-pass metabolism of imipramine and desipramine: impact of the sparteine oxidation phenotype. Clin Pharmacol Ther 43:400-406.

Cashman JR and Zhang J (2006) Human flavin-containing monooxygenases. Annu Rev Pharmacol Toxicol 46:65-100.

Chen Y, Zane NR, Thakker DR, and Wang MZ (2016) Quantification of flavin-containing monooxygenases 1,3 , and 5 in human liver microsomes by UPLC-MRM-based targeted quantitative proteomics and its application to the study of ontogeny. Drug Metab Dispos 44: 975-983.
Chiu CC, Lane HY, Huang MC, Liu HC, Jann MW, Hon YY, Chang WH, and Lu ML (2004) Dose-dependent alternations in the pharmacokinetics of olanzapine during coadministration of fluvoxamine in patients with schizophrenia. J Clin Pharmacol 44 $1385-1390$.

Dolphin CT, Cullingford TE, Shephard EA, Smith RL, and Phillips IR (1996) Differential developmental and tissue-specific regulation of expression of the genes encoding three members of the flavin-containing monooxygenase family of man, FMO1, FMO3 and FM04. Eur J Biochem 235:683-689.

Elshafeey AH, Elsherbiny MA, and Fathallah MM (2009) A single-dose, randomized, two-way crossover study comparing two olanzapine tablet products in healthy adult male volunteers under fasting conditions. Clin Ther 31:600-608.

Fuchs WS, Leary WP, van der Meer MJ, Gay S, Witschital K, and von Nieciecki A (1996) Pharmacokinetics and bioavailability of tamoxifen in postmenopausal healthy women. Arzneimittelforschung 46:418-422.

Garg DC, Eshelman FN, and Weidler DJ (1985) Pharmacokinetics of ranitidine following ora administration with ascending doses and with multiple-fixed doses. J Clin Pharmacol 25 437-443.

Haining RL, Hunter AP, Sadeque AJ, Philpot RM, and Rettie AE (1997) Baculovirus-mediated expression and purification of human FMO3: catalytic, immunochemical, and structural characterization. Drug Metab Dispos 25:790-797.

Hines RN and McCarver DG (2002) The ontogeny of human drug-metabolizing enzymes: phase I oxidative enzymes. J Pharmacol Exp Ther 300:355-360

Humphries H, Neuhoff S, and Gardner I (2015) Application of PBPK modelling for prediction of FMO metabolism using benzydamine as a probe for FMO3, in Proceedings of the 44th DMDG Open Meeting, Cambridge UK September 16th to 18th 2015.

Jamei M, Marciniak S, Edwards D, Wragg K, Feng K, Barnett A, and Rostami-Hodjegan A (2013) The Simcyp population based simulator: architecture, implementation, and quality assurance. In Silico Pharmacol 1:9.

Jones BC, Srivastava A, Colclough N, Wilson J, Reddy VP, Amberntsson S, and Li D (2017) An investigation into the prediction of in vivo clearance for a range of flavin-containing monooxygenase substrates. Drug Metab Dispos 45:1060-1067.

Katagiri F, Shiga T, Inoue S, Sato Y, Itoh H, and Takeyama M (2006) Effects of itopride hydrochloride on plasma gut-regulatory peptide and stress-related hormone levels in healthy human subjects. Pharmacology 77:115-121.

Koukouritaki SB and Hines RN (2005) Flavin-containing monooxygenase genetic polymorphism: impact on chemical metabolism and drug development. Pharmacogenomics 6:807-822.

Koukouritaki SB, Simpson P, Yeung CK, Rettie AE, and Hines RN (2002) Human hepatic flavincontaining monooxygenases 1 (FMO1) and 3 (FMO3) developmental expression. Pediatr Res 51:236-243.

Lin J (2015) In-vitro-in-vivo extraploation of UGT1A9-mediated clerance and application in a PBPK model for dapagliflozin, in Proceedings of the 44th DMDG Open Meeting, Cambridge UK September 16th to 18 th 2015 .

Luzon E, Blake K, Cole S, Nordmark A, Versantvoort C, and Berglund EG (2017) Physiologically based pharmacokinetic modeling in regulatory decision-making at the European Medicines Agency. Clin Pharmacol Ther 102:98-105.

McNeil JJ, Mihaly GW, Anderson A, Marshall AW, Smallwood RA, and Louis WJ (1981) Pharmacokinetics of the $\mathrm{H}_{2}$-receptor antagonist ranitidine in man. $\mathrm{Br} \mathrm{J}$ Clin Pharmacol 12 : $411-415$.

Nguyen HQ, Callegari E, and Obach RS (2016) The use of in vitro data and physiologically-based pharmacokinetic modeling to predict drug metabolite exposure: desipramine exposure in cytochrome P4502D6 extensive and poor metabolizers following administration of imipramine. Drug Metab Dispos 44:1569-1578.

Obach RS, Baxter JG, Liston TE, Silber BM, Jones BC, MacIntyre F, Rance DJ, and Wastall P (1997) The prediction of human pharmacokinetic parameters from preclinical and in vitro metabolism data. J Pharmacol Exp Ther 283:46-58.

Overby LH, Carver GC, and Philpot RM (1997) Quantitation and kinetic properties of hepatic microsomal and recombinant flavin-containing monooxygenases 3 and 5 from humans. Chem Biol Interact 106:29-45.

Raaflaub J, Haefelfinger P, and Trautmann KH (1984) Single-dose pharmacokinetics of the MAOinhibitor moclobemide in man. Arzneimittelforschung 34:80-82.

Reddy RR, Ralph EC, Motika MS, Zhang J, and Cashman JR (2010) Characterization of human flavin-containing monooxygenase (FMO) 3 and FMO5 expressed as maltose-binding protein fusions. Drug Metab Dispos 38:2239-2245.

Rodgers T, Leahy D, and Rowland M (2005) Physiologically based pharmacokinetic modeling 1: predicting the tissue distribution of moderate-to-strong bases. J Pharm Sci 94:1259-1276.

Rodgers T and Rowland M (2006) Physiologically based pharmacokinetic modelling 2: predicting the tissue distribution of acids, very weak bases, neutrals and zwitterions. J Pharm Sci 95: $1238-1257$.

Schoerlin MP, Mayersohn M, Korn A, and Eggers H (1987) Disposition kinetics of moclobemide, a monoamine oxidase-A enzyme inhibitor: single and multiple dosing in normal subjects. Clin Pharmacol Ther 42:395-404

Sjögren E, Westergren J, Grant I, Hanisch G, Lindfors L, Lennernäs H, Abrahamsson B and Tannergren C (2013) In silico predictions of gastrointestinal drug absorption in pharmaceutical product development: application of the mechanistic absorption model GI-Sim. Eur J Pharm Sci 49:679-698.

Takano A, Suhara T, Kusumi I, Takahashi Y, Asai Y, Yasuno F, Ichimiya T, Inoue M, Sudo Y, and Koyama $\mathrm{T}$ (2006) Time course of dopamine $\mathrm{D}_{2}$ receptor occupancy by clozapine with medium and high plasma concentrations. Prog Neuropsychopharmacol Biol Psychiatry 30: $75-81$.

Taniguchi-Takizawa T, Shimizu M, Kume T, and Yamazaki H (2015) Benzydamine $N$-oxygenation as an index for flavin-containing monooxygenase activity and benzydamine $\mathrm{N}$-demethylation by cytochrome $\mathrm{P} 450$ enzymes in liver microsomes from rats, dogs, monkeys, and humans. Drug Metab Pharmacokinet 30:64-69.

Tassaneeyakul W, Kittiwattanagul K, Vannaprasaht S, Kampan J, Tawalee A, Puapairoj P, Tiamkao S, Juthagridsada S, Kukongviriyapan V, and Tassaneeyakul W (2005) Steady-state bioequivalence study of clozapine tablet in schizophrenic patients. J Pharm Pharm Sci 8 $47-53$.

Traynor AM, Hewitt M, Liu G, Flaherty KT, Clark J, Freedman SJ, Scott BB, Leighton AM, Watson PA, Zhao B, et al. (2011) Phase I dose escalation study of MK-0457, a novel Aurora 
kinase inhibitor, in adult patients with advanced solid tumors. Cancer Chemother Pharmacol 67: 305-314.

Wagner C, Zhao P, Pan Y, Hsu V, Grillo J, Huang SM, and Sinha V (2015) Application of physiologically based pharmacokinetic (PBPK) modeling to support dose selection: report of an FDA public workshop on PBPK. CPT Pharmacometrics Syst Pharmacol 4:226-230.

Yeung CK, Lang DH, Thummel KE, and Rettie AE (2000) Immunoquantitation of FMO1 in human liver, kidney, and intestine. Drug Metab Dispos 28:1107-1111.

Yoon S, Lee H, Kim TE, Lee S, Chee DH, Cho JY, Yu KS, and Jang IJ (2014) Comparative steady-state pharmacokinetic study of an extended-release formulation of itopride and its immediate-release reference formulation in healthy volunteers. Drug Des Devel Ther 8 $123-128$
Zhou W, Humphries H, Neuhoff S, Gardner I, Masson E, Al-Huniti N, and Zhou D (2017) Development of a physiologically based pharmacokinetic model to predict the effects of flavincontaining monooxygenase 3 (FMO3) polymorphisms on itopride exposure. Biopharm Drug Dispos 38:389-393.

Address correspondence to: Dr. Venkatesh Pilla Reddy, AstraZeneca UK Limited, Hodgkin Building c/o B310, Cambridge Science Park, Milton Road, Cambridge, Cambridgeshire CB4 OWG, UK. E-mail: venkatesh.reddy@astrazeneca.com 